\title{
Factors related to sense of competence in family caregivers of people living with dementia in the community: A narrative synthesis
}

\author{
Jacki Stansfeld ${ }^{1,2}$, Nadia Crellin ${ }^{1,2}$, Martin Orrell ${ }^{3}$, Jennifer Wenborn ${ }^{1,2}$, Georgina \\ Charlesworth $^{2,4}$, and Myrra Vernooij-Dassen ${ }^{5}$
}

${ }^{1}$ Division of Psychiatry, University College London, Maple House, 149 Tottenham Court Road, London, W1T 7BN, UK ${ }^{2}$ Research and Development, North East London NHS Foundation Trust, Goodmayes Hospital, IG3 8XJ, UK ${ }^{3}$ Institute of Mental Health, University of Nottingham, Triumph Road, Nottingham, NG7 2TU, UK ${ }^{4}$ Department of Clinical, Educational and Health Psychology, University College London, 1-19 Torrington Place, London, WC1E 6BT, UK ${ }^{5}$ Radboud University Medical Centre, Postbus 9101, 6500 HB Nijmegen, The Netherlands

\section{Abstract}

Objectives-Sense of competence defines a caregiver's feeling of being capable to manage the caregiving task and is an important clinical concept in the caregiving literature. The aim of this review was to identify the factors, both positive and negative, associated with a caregiver's perception of their sense of competence.

Design-A systematic review of the literature was conducted, retrieving both quantitative and qualitative papers from databases PsycINFO, CINAHL, EMBASE and Medline. A quality assessment was conducted using the STROBE and CASP checklists and the quality rating informed the inclusion of papers ensuring the evidence was robust. Narrative synthesis was employed to synthesise the findings and to generate an updated conceptual model of sense of competence.

Results-Seventeen papers were included in the review, all of which were moderate to high quality. These included 13 quantitative, three mixed-methods and one qualitative study. Factors associated with sense of competence included: behavioural and psychological symptoms of dementia (BPSD), caregiver depression, gratitude and the ability to find meaning in caregiving.

Conclusions-The results of this review demonstrate that both positive and negative aspects of caring are associated with caregiver sense of competence. Positive and negative aspects of

Corresponding Author: Jacki Stansfeld, Division of Psychiatry, University College London, Maple House, 149 Tottenham Court Road, London, W1T 7NF, j.stansfeld@ucl.ac.uk, Telephone: 03005551200 ext 64491 / 07834392294.

Conflict of interest declaration

The authors confirm there is no conflict of interest.

Description of authors' roles

J. Stansfeld designed the review, conducted the searches, screening, quality appraisal and drafted the manuscript. N. Crellin was the second reviewer, screening the full texts for inclusion/exclusion and conducting a secondary quality appraisal on all identified full-text papers. J. Wenborn, G. Charlesworth, M. Orrell \& M. Vernooij-Dassen supervised, oversaw the review and contributed to the manuscript. All authors agreed to the final version prior to submission. 
caregiving act in tandem to influence caregiver perception of their competence. The proposed model of sense of competence aims to guide future research and clinical interventions aimed at improving this domain but requires further testing, as due to the observational nature of the include papers, the direction of causality could not be inferred.

\section{Keywords}

Dementia; Alzheimer's disease; carers; competency

\section{Introduction}

Dementia is a progressive and complex condition in which much of the support is provided

by family caregivers (Alzheimer's Society, 2014). As dementia progresses, the person living with dementia may need increasing support from their family caregiver, who may in turn experience a feeling of increased burden, depression or stress (Cooper et al., 2007; Crespo et al., 2005; Van der lee et al., 2014). Previous literature has often focused on the stress process and burden in care, detailing the emotional and physical burden of caregiving, which can increase the risk of both physiological and mental illness in the caregiver (Richardson, et al., 2013; Cupidi et al., 2012). More recently, however, studies are beginning to investigate the positive aspects of caring and protective factors against the stress of caregiving, such as sense of competence.

Sense of competence is defined as a state related to the caregiver's feeling of being capable to manage the caregiving role (Vernooij-Dassen et al., 1996). Sense of competence is an important clinical concept as research findings show that if a family caregiver responds negatively to the demands of caregiving it will increase the risk that the person with dementia moves to residential care and increase the probability of caregiver ill health (Spijker et al., 2011). A higher sense of competence has been linked with lower levels of burden (Kurz et al., 2003) and a reduced risk that the person with dementia will enter residential care (Borsje et al., 2016).

Consideration must be given to the overlap between the concepts of self-efficacy and sense of competence. Whilst sense of competence is a global domain, relating not only to the caregiver's ability to perform each task but also how important each task is perceived to be, and how well the care is received by the person with dementia, self-efficacy has been argued to be domain-specific, referring to "beliefs in one's capabilities to organise and execute the course of action required to produce given attainments", (Bandura, 1997, pp.3). Despite this, further work to understand, measure and conceptualise sense of competence is clearly required. For the purposes of this review, the definition and conceptualisation of sense of competence from Vernooij-Dassen et al. (1996) described previously will be employed.

The existing theoretical model and subsequent research into sense of competence (VernooijDassen et al., 1996) demonstrated a relationship between sense of competence and factors related to the personality of the caregiver, such as neuroticism, perceived social support and also person with dementia factors such as apathy and behavioral disturbances (see Figure 1). However, the impact of positive appraisal of the caring situation and positive personality traits on sense of competence were not explored. Although sense of competence is 
increasingly being recognized as an important concept in international research for this population (Moniz-Cook, 2008; Lau et al., 2015), little further work has been done since the original conceptual model and to the authors' knowledge, no systematic review has been undertaken to address this gap. Knowledge of how to improve a caregiver's feelings of competence and the factors that relate to this concept can inform the design of support services and interventions to enable them to care for the person with dementia in the community for longer.

Therefore, the aim of this review was to explore and develop further the conceptual model of factors related to sense of competence. Specifically, this review addressed the following research questions: (1) What caregiver factors are related to sense of competence? (2) Are positive aspects of caring related to sense of competence? (3) What person with dementia factors are related to sense of competence?

\section{Method}

The search terms for this review were developed through scoping of previous literature in order to guide the inclusion/ exclusion of papers in the systematic review. Scoping of previous literature was conducted by searching for and reading relevant published papers related to sense of competence to ensure familiarity with the topic and to generate search terms.

\section{Search Strategy}

A systematic search was conducted in Medline, PsycINFO, CINAHL and EMBASE electronic databases. These databases were chosen due to their relevance to the aims and topic of this review.

Search terms were divided into three categories: sense of competence, family caregiver and dementia. The search terms were combined ('sense of competence' AND 'family caregiver 'AND dementia), with truncations of terms used where appropriate. Search terms for sense of competence included overlapping constructs such as self-efficacy and mastery in order to ensure that all possible relevant papers were identified: 'sense of competence' OR 'competence' 'perceived competence' OR 'mastery' OR ‘self-efficacy' OR 'burden' OR ‘SCQ' OR ‘capability'.

Search terms for family carer were: 'family carer' OR 'caregiver' OR 'caring' OR 'care' OR 'carer' OR 'relative' OR 'friend' OR 'spouse' OR 'supporter' OR 'support'. Search terms for dementia were: 'cognitive impairment' OR 'Alzheimer' OR 'senile' OR 'dementia' OR 'demented' OR 'MCI'. The terms 'cognitive impairment' and 'MCI' were included in order to ensure that studies with mixed populations were captured. Hand searching of references and citations of all papers included at the full-text stage was conducted to ensure a thorough search, however; no additional papers were identified. The principles outlined by the PRISMA group were followed (Moher et al., 2009).

\section{Inclusion Criteria}

- Studies published in English only, unless a translation was available 
- $\quad$ Studies published from the earliest date available on each database to November 2016 (date of the search). No papers preceding 1980 were identified.

- Qualitative or quantitative studies. Quantitative studies were required to include a standardized measure of sense of competence

- $\quad$ Studies examining the relationship between or factors affecting sense of competence. This did not have to be the primary aim of the study.

\section{Exclusion Criteria \\ - $\quad$ Systematic review papers \\ - $\quad$ Studies conducted with family carers of people living in residential care \\ - Intervention studies, as these only investigate the impact of an intervention on sense of competence rather than factors relating to this construct. \\ - Qualitative or quantitative studies deemed to be of 'poor quality' as assessed by the STROBE and CASP checklists.}

\section{Data Extraction}

Papers were exported to Mendeley Referencing Software and checked for duplicates. Titles were screened and irrelevant titles that did not meet the inclusion criteria were excluded. Titles where it was not possible to determine if they should be included were retained in order to screen in further detail. Abstracts were then screened and for those identified as relevant the full text was downloaded. The final eligibility decision was made by reading the full text. In cases where more information was needed to determine eligibility, or when there was difficulty obtaining the full text, the study authors were contacted.

Once the full-texts had been obtained, two authors (JS \& NC) independently reviewed a randomly chosen sub-set of five papers against the inclusion/exclusion criteria. Data relating to the design (qualitative/quantitative) methodology, author, year, sample population, sample size, measures used, analysis and findings was extracted onto a data extraction database for these five papers. A consensus meeting was held to cross-check data extraction. In instances in which there was disagreement regarding whether a paper should be included, of which there was one, discussions were held until a consensus was reached. As agreement was high, the remaining full-texts were randomly divided between the two researchers for screening against inclusion/exclusion criteria and a further consensus meeting was held before the two data extraction databases were merged. JS completed a quality assessment checklist for all included full-text papers and a consensus meeting was held with NC to discuss scores. At this stage, any disagreements regarding quality assessment scoring were discussed and quality assessment scores were agreed. A flow chart of the systematic review process is illustrated in Figure 2.

\section{Quality Assessment}

The methodological quality of the quantitative studies was evaluated using the 22 item STROBE checklist (von Elm et al., 2007), which was developed to assess the methodological quality of the reporting of observational research. This tool contains 22 
items. The authors scored each of the items according to whether it had been met; no (scored zero) and yes (scored one point). Item scores for each study were summed to create a total quality score out of 22 and this was represented as a percentage of the total possible score. The researchers devised cut-off scores in order to allow ease of description of the quality of each of the studies. Studies that scored below 50\% were considered to be of 'poor quality' and were excluded from the review. Studies scoring 50-74\% were rated to be of moderate quality and studies scoring above $75 \%$ were graded as high quality. Both moderate and high quality studies were included in this review as these were considered sufficiently robust for the synthesis.

\section{Qualitative papers}

Qualitative studies were assessed for quality using the 10-item Critical Appraisal Skills Programme (CASP) qualitative research appraisal criteria (Public Health Resource Unit, 2006). This tool scores methodological quality using 10 items, with response items scored as: meeting the criteria (score of 1), unable to tell (score of 0 ) or not meeting the criteria (score of 0 ). The scores are summed to produce an overall quality score. Only studies rating more than 5 out of $10(50 \%)$ were included in the review.

\section{Mixed methods papers}

Mixed methods studies were subject to both the STROBE and CASP quality assessment and had to meet the predefined standards for both.

\subsubsection{Design}

A narrative synthesis design was employed for this review, using guidance from a published protocol (Popay et al., 2006). This approach allows the combination of qualitative, quantitative and mixed-methods studies to reach a conclusion based on interpretation of the text.

\section{Narrative Synthesis Methodology}

There are four steps in narrative synthesis, as follows:

1. Development of a theory

Stage one involved scoping of the literature, before conducting the search, to guide the research question and the inclusion/exclusion criteria. Current theoretical models of the caregiver stress-coping process (Sorensen \& Conwell, 2011), and the existing theoretical model of sense of competence were used to guide the review (Vernooij-Dassen et al., 1996).

2. Development of a preliminary synthesis of the findings

Stage 2 involved grouping the papers according to design (qualitative/ quantitative). Data were extracted from each of the studies, clustered by methodology (observational, mixed methods and qualitative studies) and then tabulated to enable comparison.

3. Exploring the relationships in the data within and between studies 
The tabulated summary was used to explore the relationships within the data. Similarities and differences between outcomes in the studies were considered. Factors that may have influenced heterogeneity in findings, such as study design, methodological quality or characteristics were explored.

4. Assessing the robustness of the synthesis

For stage 4, the methodological quality of each included study was assessed using two standardized tools (STROBE and CASP quality assessment checklists) in order to ensure all included evidence was of good quality, as this would affect the robustness of the synthesis. At all stages of the review, the robustness of the methods and synthesis were evaluated with an informal critical reflection, looking back over the methodology and review process to identify any limitations or biases that may have impacted the findings of the review. These are reported further in the discussion section where applicable.

The narrative synthesis approach may be adopted to generate a conceptual map of findings (Popay et al., 2006). The authors used the results of this review to generate a conceptual model to reflect the findings. In order to generate a model, findings were grouped by concept where applicable, for example, BPSD and coping style. The relationship of these concepts with sense of competence was considered and a model was drawn according to the findings, to include all factors related to sense of competence identified in the included studies. A consensus meeting was held between three authors (JS, MO, MVD) in order to agree and finalize the conceptual map according to the literature and to incorporate these with the existing model of sense of competence (Vernooij-Dassen et al., 1996).

\section{Results}

The initial search returned 9370 papers after duplicates were removed, of which 49 potential full-text publications were extracted. At the initial stage, the main reason for exclusion of articles were that they were not related to sense of competence or that they were not conducted in a population of dementia caregivers. After reviewing the full articles, 17 met the inclusion criteria. Thirteen were quantitative studies, three were mixed methodology and one was qualitative. The main reason for exclusion of papers were that they were intervention studies (14 excluded) or that they were not conducted in a population of family caregivers of people with dementia living in the community (seven excluded). Figure 2 reports these figures in more detail. Following quality assessment, no further studies were excluded. Complete quality assessment scores are reported in Tables 1 and 2.

\section{Stage 2: Preliminary synthesis of the findings}

Detail on included studies, such as design, analysis and sample size can be found in Tables 1 \& 2. The articles were largely from the Netherlands $(n=8)$, but also from the UK $(n=3)$, USA $(n=2)$, Taiwan $(n=1)$, Belgium $(n=1)$, Singapore $(n=1)$ and Canada $(n=1)$. In the included articles, the majority were cross-sectional $(n=9)$ or longitudinal $(n=5)$ studies. Two of the mixed-methods articles reported results from interview studies alongside quantitative findings and one reported results from interviews and focus groups. One article reported 
mixed-methods results from a case study. The total number of patients in all included studies was 2463 , with a mean of 144 and a range of $1-447$. For quantitative studies the total number of participants was 2277, with a mean of 190 and a range of $56-447$. For the mixed-methods studies, the total number of participants was 150 , with a mean of 50 and a range of 1-99. In the one qualitative paper (Peacock et al., 2010), there were 39 participants.

\section{Stage 3: Exploring relationships within and between studies Factors related to sense of competence}

Factors related to sense of competence identified in the systematic review were related to both person with dementia and caregiver factors. All included studies were of moderate to high quality. The quantitative papers were mostly of high quality (54\%) and the remainder of the papers were of moderate quality $(46 \%)$. The mixed methods papers all scored moderately on the STROBE checklist for the quantitative methodology and high on the CASP checklist for the qualitative methodology. The one qualitative study scored high on the CASP checklist. The evidence presented in the included papers was considered robust enough to be included in the synthesis.

The mean age of caregivers across the included studies was 61.6. All of the included studies were conducted in the community, with caregivers caring for a person with dementia who was a family member or friend.

\section{Caregiver factors and sense of competence}

Demographic factors-Compared with male caregivers, being a female caregiver living with a person with dementia predicted a lower sense of competence in a prospective longitudinal study (Vernooij-Dassen et al., 1997). In addition, the age of the caregiver was associated with sense of competence, with younger caregivers (50-70 years of age) reporting a lower sense of competence than older caregivers ( $>70$ years of age) in a fixed effects model (Borsje et al., 2016). Borsje et al. (2016) also found that adult child caregivers had a higher sense of competence than spouses and that a lower sense of competence was associated with the likelihood that the person with dementia would be admitted to a longterm care facility. Millenaar et al. (2015) found that there was no difference between caregivers of young onset and late onset dementia over the course of a two year study.

Negative aspects of caregiving-Sense of competence was negatively correlated with symptoms of depression in the caregiver (Lau et al., 2015), although this was only a small relationship. This indicates that the higher the depressive symptoms, the lower the sense of competence the caregivers felt. In the qualitative literature, a theme of hopelessness emerged, which was associated with a decrease in sense of competence. This theme included a fear of not knowing what to do and how this might impact the future. "I don't know how I can help him. I'm tired and I have back problems because of all the things I have to do myself at the moment. I am afraid for the future" (Graff et al., 2006, pp. 513).

Positive aspects of caregiving-Despite this, sense of competence seemed also to be related to positive aspects of caregiving. Caregiver competence was found to moderately 
correlate with self-efficacy for managing dementia in a validation study (Fortinsky et al., 2002). Other positive aspects of caregiving such as gratitude were also moderately related to caregiving competence (Lau et al., 2015). This was reflected in the qualitative literature, as caregivers were able to derive confidence in themselves and learnt about their strengths from the caregiving experience: "I have learned a lot about my own strengths - having more confidence in myself to make major decisions alone" (Narayan et al., 2001, pp 24). In addition, the ability to find meaning in caregiving (Quinn et al., 2012a; Quinn et al., 2012b) was moderately related to sense of competence. In terms of coping strategies, the use of emotion-focused (Lau et al., 2015) coping was moderately related to sense of competence. In a regression analysis, religious involvement positively predicted sense of competence (Vernooij-Dassen et al., 1997), whereas the use of negative caregiving strategies was moderately related to a lower sense of competence (Tan et al., 2013). In a longitudinal study, there was a significant difference between caregivers who used a supporting strategy and those who used a non-adaptive strategy, with carers using a supportive coping strategy scoring higher on sense of competence. From these studies, it seems that the ability to identify positive aspects of caregiving, such as finding meaning and gratitude in the caregiving role may act as a protective factor for sense of competence against the negative outcomes in caregiving, such as depression.

\section{Person with dementia factors and sense of competence}

Sense of competence was moderately to strongly negatively associated with symptoms of BPSD of the person with dementia in three studies (Borsje et al., 2016; Feast et al., 2016; Van der lee et al., 2015) and was also found to mediate BPSD frequency and BPSD related distress in a structural equation model (Feast et al., 2016). Sense of competence was significantly and negatively associated with reactivity to BPSD and caregiver guilt, which were both moderate associations (Feast et al., 2016). The qualitative literature reflected this finding, as it emerged that caregivers found their sense of competence was challenged by BPSD, however, finding a way to deal with these behaviours gave them a sense of accomplishment and pride which in turn improved their sense of competence "It's amazing the difference knowing what to do, and how to do it" (Peacock et al., 2010, pp 650). In a longitudinal study, sense of competence, severity of neuropsychiatric symptoms in the person with dementia and high caregiver dominance scores were significant predictors of emotional distress. (Van der lee et al., 2015).

Severity of dementia was indirectly and moderately related to sense of competence through the caregiver's reports of apathetic behaviour of the person with dementia in a path analysis model (Vernooij-Dassen et al., 1996). Therefore, the more severe the dementia, the more likely the caregiver was to report apathetic behaviour in the person with dementia and consequently, have a lower sense of competence. A longer duration of dementia was related to sense of competence, with decreases in sense of competence positively correlated with duration of dementia (Vernooij-Dassen et al., 2003). However, in another study (Kurz et al., 2003), sense of competence scores for caregivers of people with severe dementia was found to be comparable to caregivers of patients with mild to moderate dementia, although these statistics were only descriptive. The results of this review have contributed to an updated model of sense of competence (Figure 3). 


\section{Discussion}

This review is the first to systematically explore the factors related to sense of competence from a theoretical perspective. There were 17 studies included in this mixed-methods review. The majority of the studies found that situational, demographic and personality characteristics of the caregiver related to sense of competence. In addition, person with dementia factors such as BPSD and duration of dementia were associated with sense of competence. Sense of competence mediated the relationship between BPSD frequency and distress, indicating that whilst sense of competence is influenced by factors such as BPSD, it may also serve as a protective factor against the negative outcomes of caring such as depression and distress if the carer can identify positive aspects of caring such as finding meaning in their role.

The findings identified in this review presented an opportunity to update the existing model of sense of competence in order to address conceptual gaps. The use of narrative synthesis enabled the combination of both qualitative and quantitative research to contribute to this model. The previously hypothesized theoretical model (Vernooij-Dassen et al., 1996) did not include association of sense of competence with the positive aspects of caregiving, such as gratitude, meaning and self-efficacy alongside the association with the negative outcomes of caregiving. The proposed updated model might therefore contribute to a better understanding of the positive and negative aspects of the caregiving experience and how they relate to sense of competence.

\section{Critical reflection (narrative synthesis stage 4)}

Critical reflection was undertaken throughout the review in order to identify any biases or limitations that could impact the review, according to published guidance (Popay et al., 2006). A formal approach to testing the robustness of the synthesis was not undertaken as the critical reflection is not a linear process, however; the authors recognize that potential limitations of this review may therefore be missed.

This review had well defined and broad inclusion criteria, which were identified from existing literature and sense of competence theory. However, as with all systematic reviews, the findings here rely on the quality of the included studies and the authors note that the findings were not weighted based on the methodological quality, which could be a limitation. This decision was made because narrative synthesis research takes a textual approach to presenting and combining evidence from different methodologies, making it difficult to fairly weight the evidence, as no single indicator of methodological quality was considered important for this review, rather the total score informed inclusion of studies. All of the included studies were of good methodological quality and therefore the results were considered equally.

Consideration must be given to the potential for measurement error to influence the findings of the review. Narrative synthesis combines results from included papers without re-analysis and therefore if measurement error is present in the included quantitative studies, it will carry forward to the findings of this review. In addition, in qualitative or mixed methods studies, if there is any bias present in the qualitative analysis, this could affect the findings of 
the review. Despite this, the included qualitative and mixed methods papers were all of high methodological quality and had rigorous analyses, so it is anticipated that the impact of bias is limited.

This review combined the results of several sense of competence outcome measures, which may present a methodological limitation due to differences in operationalization between the scales. The conceptualization of sense of competence is an existing methodological issue as it overlaps with the concept of self-efficacy and is often poorly defined. To minimize the impact of this on the review, the authors only included studies where sense of competence had been measured with a valid and standardized outcome measure. In addition, all included studies employed definitions of sense of competence reflecting that conceptualized by Vernooij-Dassen et al., (1996). Despite this, the authors acknowledge that further work is required to fully conceptualize sense of competence and ensure that it is measured consistently between studies.

The majority of the studies included within the review were cross-sectional and reported correlations, therefore this study could only infer a relationship between sense of competence and the factors identified, rather than infer causality or identify change. Additionally, a number of studies were excluded from this review that may have contained potentially relevant results. These studies were excluded as they contained mixed populations of caregivers of people living with dementia both in the community and in residential care and did not separate the results by population. This review was concerned with factors related to sense of competence only in family caregivers of people living with dementia in the community and therefore the studies were excluded. It is recommended that authors endeavor to report the results from different populations (e.g. community and residential care) separately in research studies rather than pooling populations together, which could impact the results.

\section{Future Research}

Limited qualitative research investigating sense of competence was identified in this review. Qualitative exploration of what sense of competence means to family caregivers of people living with dementia, and their perceptions of how it can be improved would give a deeper understanding of what caregivers themselves feel determines their sense of competence. The theoretical model developed by the review authors was based on conceptual mapping of findings from 17 studies and has not been tested and therefore it is recommended that the model be statistically examined in both cross-sectional and longitudinal research. Despite this, the proposed theoretical model can contribute to the development of interventions aimed at increasing caregiver sense of competence.

\section{Conclusion}

This is the first review to investigate the factors related to sense of competence using a narrative synthesis approach. The proposed model indicates that both positive and negative aspects of caregiving can impact a caregiver's sense of competence. It is likely that the positive and negative aspects of caregiving act in tandem to influence caregiver perception of their competence, reflecting the "mixed valence" of caregiving proposed in previous 
literature (Lawton et al. 1991, p.182). This review has clinical relevance as it gives an insight into the factors that relate to sense of competence, which may aid the design of interventions seeking to support family caregivers of people with dementia. It is imperative that gaps in current conceptual models and literature are addressed in order to further our understanding of this domain.

\section{Acknowledgements}

This systematic review was conducted as part of the Valuing Active Life in Dementia (VALID) research programme funded by the National Institute for Health Research (NIHR) under its Programme Grants for Applied Research scheme (RP-PG0610-10108). The VALID research team acknowledges the support of the National Institute for Health Research Clinical Research Network. The views expressed are those of the author(s) and not necessarily those of the NHS, the NIHR or the Department of Health.

\section{References}

Alzheimer's Society. Dementia 2014: Opportunity for change. Alzheimer's Society; London, UK: 2014.

Bandura, A. Self-efficacy - The exercise of control. New York, NY: W.H. Freeman \& Co.; 1997.

Borsje P, Hems MA, Lucassen PL, Bor H, Koopmans RT, Pot AM. Psychological distress in informal caregivers of patients with dementia in primary care: course and determinants. Family Practice. 2016; 33:374-381. [PubMed: 26969508]

Cooper C, Balamurali TB, Livingston G. A systematic review of the prevalence and covariates of anxiety in caregivers of people with dementia. International Psychogeriatrics. 2007; 19:175-195. [PubMed: 17005068]

Crellin NE, Orrell M, McDermott O, Charlesworth G. Self-efficacy and health-related quality of life in family carers of people with dementia: a systematic review. Aging \& Mental Health. 2014; 18:954969. [PubMed: 24943873]

Crespo M, Lopez J, Zarit SH. Depression and anxiety in primary carers: a comparative study of carers of demented and nondemented older persons. International Journal of Geriatric Psychiatry. 2005; 20:591-592. [PubMed: 15962352]

Cupidi C, Realmuto S, Coco GL, Cinturino A, Talamanca S, Arnao V, et al. Coco DL. Sleep quality in caregivers of patients with Alzheimer's disease and Parkinson's disease and its relationship to quality of life. International psychogeriatrics. 2012; 24(11):1827-1835. [PubMed: 22652066]

De Vugt ME, Stevens F, Aalten P, Lousberg R, Jaspers N, Winkens I, Jolles J, Verhey FR. Do caregiver management strategies influence patient behaviour in dementia? International Journal of Geriatric Psychiatry. 2004; 19:85-92. [PubMed: 14716704]

Feast A, Orrell M, Russell I, Charlesworth G, Moniz-Cook E. The contribution of caregiver psychosocial factors to distress associated with behavioural and psychological symptoms in dementia. Geriatric Psychiatry. 2016; 32:76-85. [PubMed: 26891463]

Fortinsky RH, Kercher K, Burant CJ. Measurement and correlates of family caregiver self-efficacy for managing dementia. Aging and Mental Health. 2002; 6:153-160. [PubMed: 12028884]

Graff MJ, Vernooij-Dassen MJ, Zajec J, Rikkert MG, Hoefnagels WH, Dekker J. How can occupational therapy improve the daily performance and communication of an older patient with dementia and his primary caregiver? Dementia. 2006; 5:502-532.

Kruz X, Scuvee-Moreau J, Vernooij-Dassen MJ, Dresser A. Cognitive impairment, dementia and quality of life in patients and caregivers. Acta Neurologica Belgica. 2003; 103:24-34. [PubMed: 12704980]

Lau BH, Cheng C. Gratitude and coping among familial caregivers of persons with dementia. Aging and Mental Health. 2015; 27:1-9.

Lawton MP, Moss M, Kleban MH, Glicksman A, Rovine M. A two-factor model of caregiving appraisal and psychological well-being. Journal of Gerontology. 1991; 46:181-89. 
Lazarus, RS, Folkman, S. Stress, appraisal, and coping. New York: Springer publishing company; 1984.

Millenaar JK, de Vugt ME, Bakker C, Pijnenburg YA, Koopmans RT, Verhey FR. The impact of Yong Onset Dementia on informal caregivers compared with Late Onset Dementia: Results from the NeedYD Study. American Journal of Geriatric Psychiatry. 2015; 24:467-474. [PubMed: 26560507]

Narayan S, Lewis M, Tornatore J, Hepburn K, Corcoran-Perry S. Subjective responses to caregiving for a spouse with dementia. Journal of Gerontological Nursing. 2001; 27:19-28.

Moher D, Liberati A, Tetzlaff J, Altman DG, Prisma Group. Preferred reporting items for systematic reviews and meta-analyses: the PRISMA statement. PLoS medicine. 2009; 6(7)

Peacock S, Forbes D, Markle-Reid M, Hawranik P, et al. The positive aspects of the caregiving journey with dementia: Using a strengths-based perspective to reveal opportunities. Journal of Applied Gerontology. 2010; 29:640-659.

Popay, J, Roberts, H, Sowden, A, Petticrew, M, Arai, L, Rodgers, M, Britten, N, Roen, K, Duffy, S. Guidance on the conduct of narrative synthesis in systematic reviews. A product from the ESRC methods programme Version,1. 2006. 92

Public Health Resource Unit. The Critical Skills Appraisal Programme: making sense of evidence. Public Health Resource Unit; England: 2006. Retrieved from: http://www.casp-uk.net/

Quinn C, Clare L, McGuinness T, Woods R. The impact of relationships, motivations and meanings on dementia caregiving outcomes. International Psychogeriatrics. 2012a; 24:1816-1826. [PubMed: 22652014]

Quinn C, Clare L, Woods R. What predicts whether caregivers of people with dementia find meaning in their role? International Journal of Geriatric Psychiatry. 2012b; 27:1195-1202. [PubMed: 22334416]

Richardson TJ, Lee SJ, Berg-Weger M, Grossberg GT. Caregiver health: health of caregivers of Alzheimer's and other dementia patients. Current psychiatry reports. 2013; 15:367. [PubMed: 23712718]

Shea BJ, Reeves BC, Wells G, Thuku M, Hamel C, Moran J, Moher D, Tugwell P. AMSTAR 2: a critical appraisal tool for systematic reviews that include randomised or non-randomised studies of healthcare interventions, or both. BMJ. 2017; 328:j4008.

Sorensen S, Conwell Y. Issues in dementia caregiving: Effects on mental and physical health, intervention strategies, and research needs. American Journal of Geriatric Psychiatry. 2011; 19:491-496. [PubMed: 21502853]

Spijker A, Wollersheim H, Teeresnstra S, Graff M, Adang E, Verhey F, Vernooij-Dassen M. Systematic care for caregivers of patients with dementia: a multicentre, cluster randomized controlled trial. American Journal of Geriatric Psychiatry. 2011; 19:521-531. [PubMed: 21358385]

Tan L, Yap P, Ng WY, Luo N. Exploring the use of the Dementia Management Strategies Scale in caregivers of persons with dementia in Singapore. Aging and Mental Health. 2013; 17:935-941. [PubMed: 23410096]

Van der Lee J, Bakker T, Duivenvoorden H, Droes R. Do determinants of burden and emotional distress in dementia caregivers change over time? Aging and Mental Health. 2015; 21:232-240. [PubMed: 26586266]

Vernooij-Dassen MJ, Persoon JM, Felling AJ. Predictors of sense of competence in caregivers of demented persons. Social science \& medicine. 1996; 43:41-49. [PubMed: 8816009]

Vernooij-Dassen M, Felling A, Persoon J. Predictors of change and continuity in home care for dementia patients. International Journal of Geriatric Psychiatry. 1997; 12:671-677. [PubMed: 9215952]

Vernooij-Dassen M, Van Hoult H, Hund K, Hoefnagles W, Grol R. Information for dementia patients and their caregivers: what information does a memory clinic pass on, and to whom? Aging and Mental Health. 2003; 7:34-38. [PubMed: 12554313]

Von Elm E, Altman DG, Egger M, Pocock SJ, Gøtzsche PC, Vandenbroucke JP, Strobe Initiative. The Strengthening the Reporting of Observational Studies in Epidemiology (STROBE) statement: guidelines for reporting observational studies. PLoS medicine. 2007; 4(10):e296. [PubMed: 17941714] 


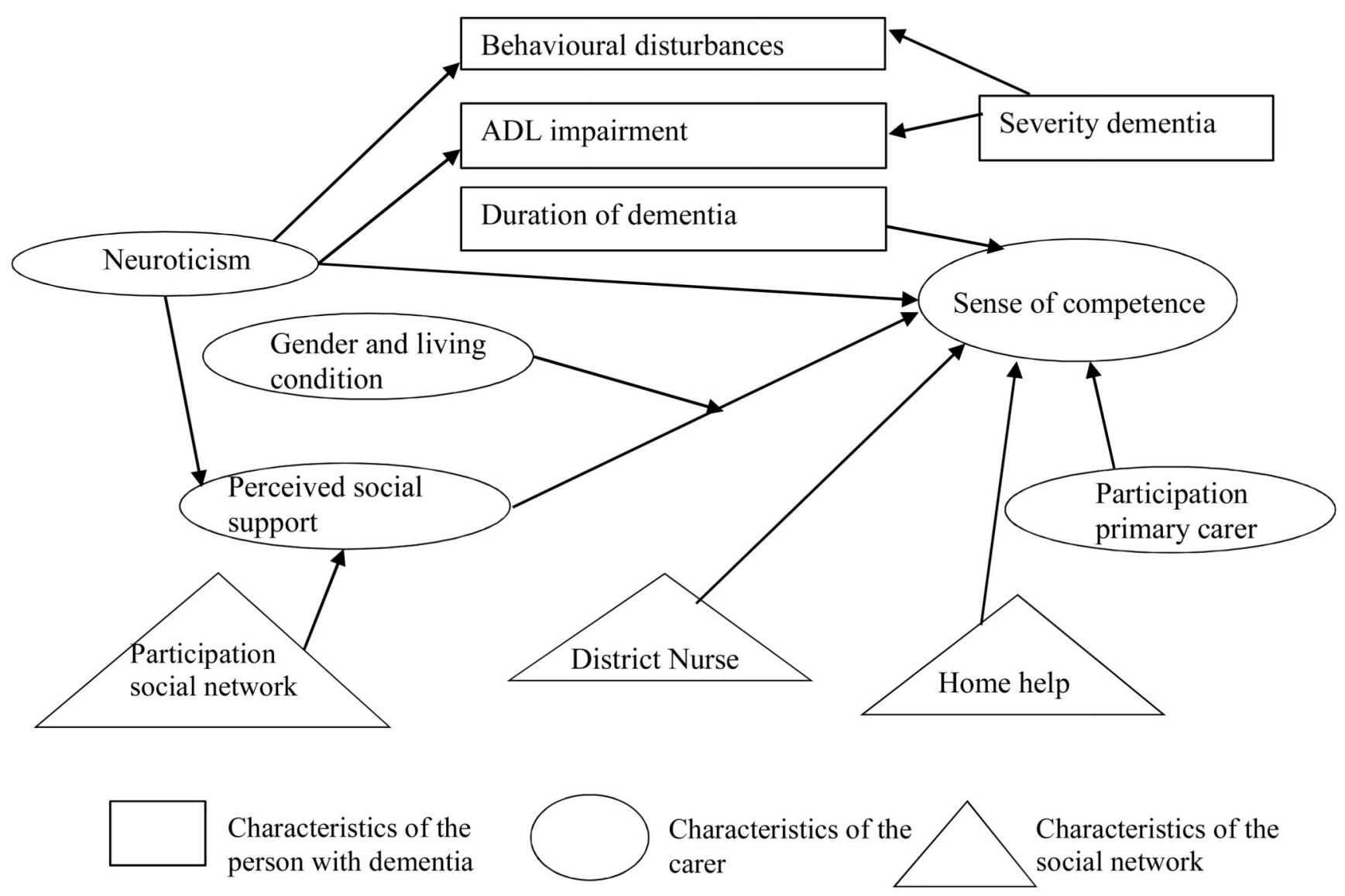

Figure 1. Predictors of sense of competence (model of sense of competence, adapted from Vernooij-Dassen et al., 1996) 


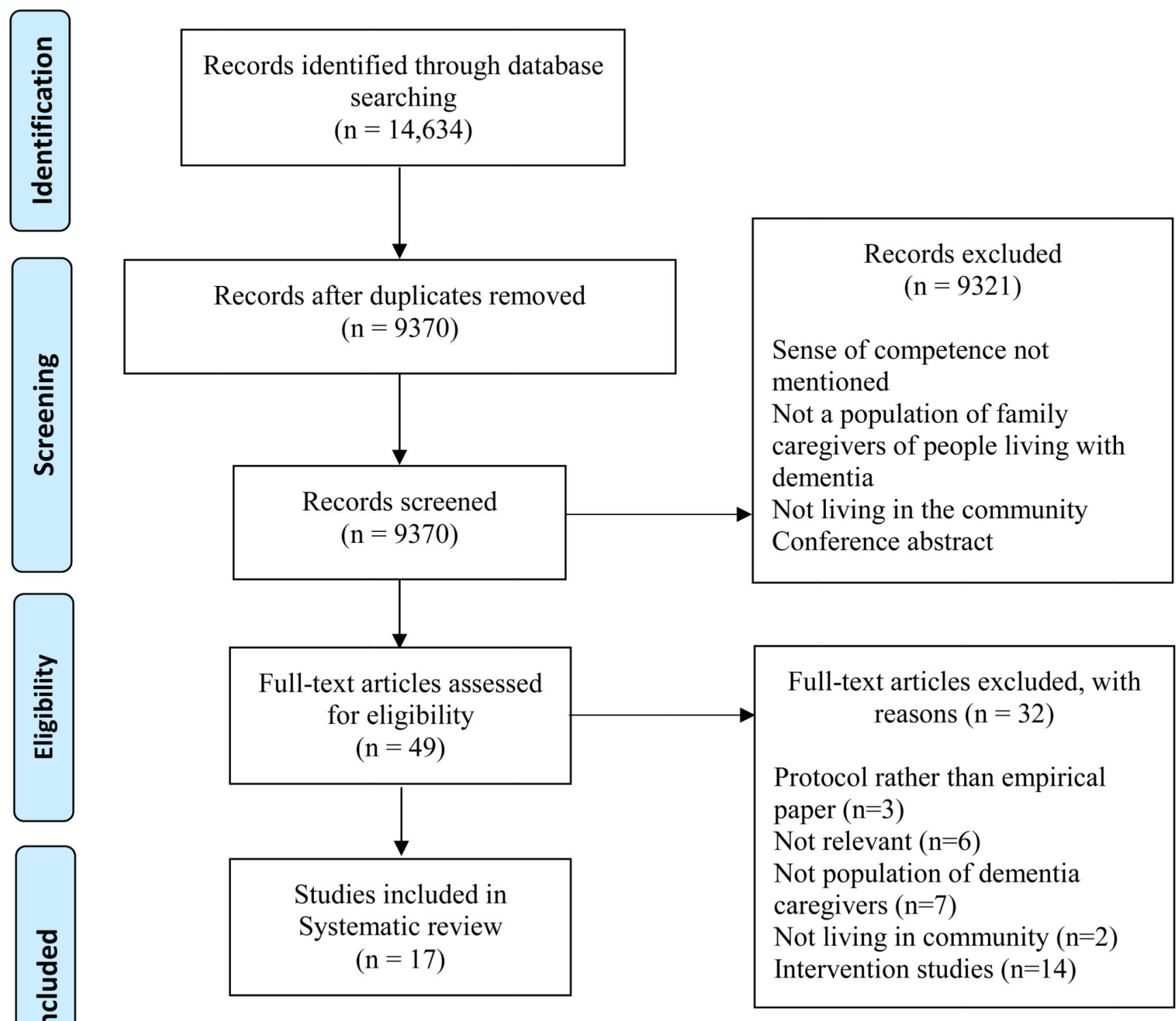

Figure 2. Process of the systematic review search 


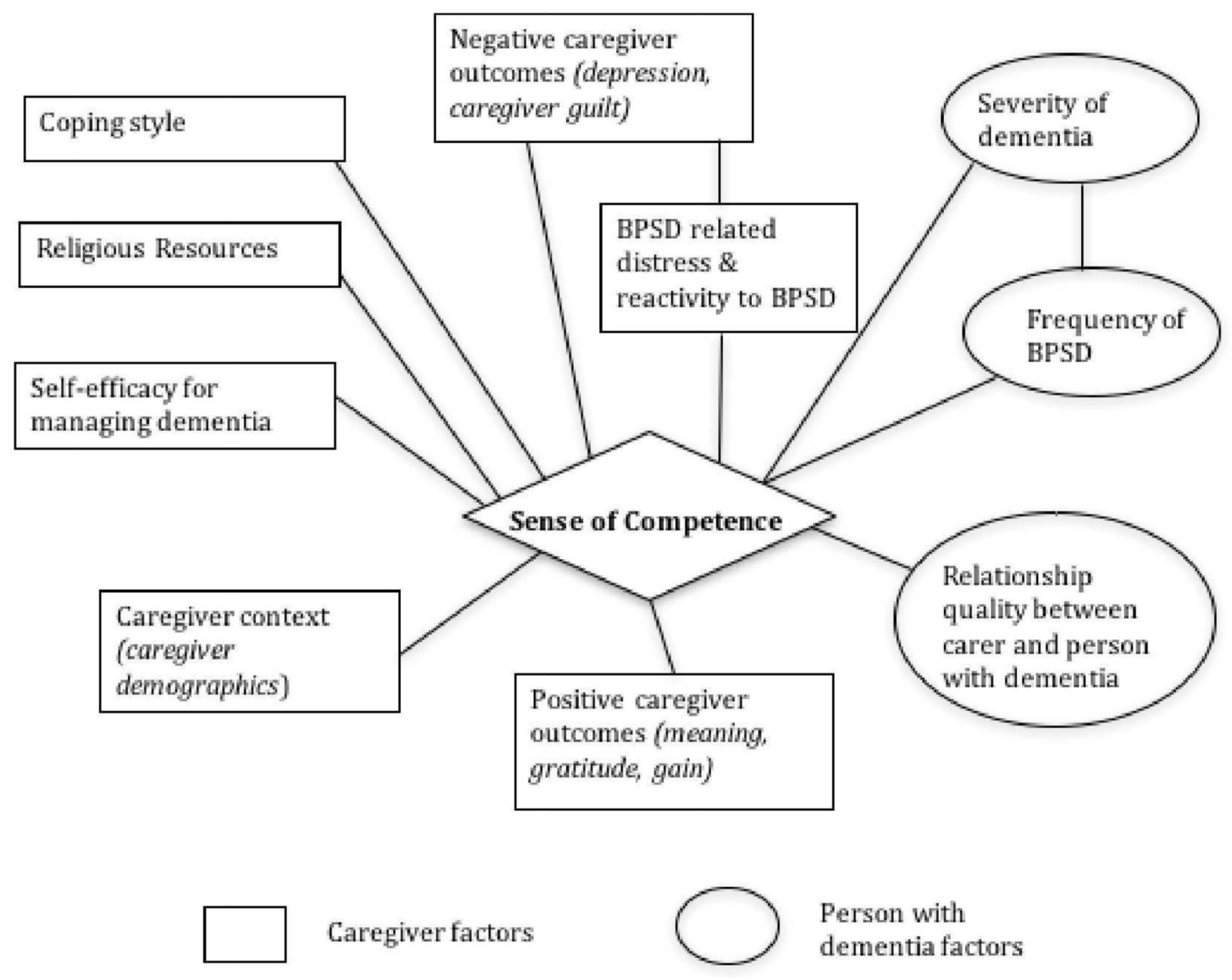

Figure 3. Updated model of sense of competence 


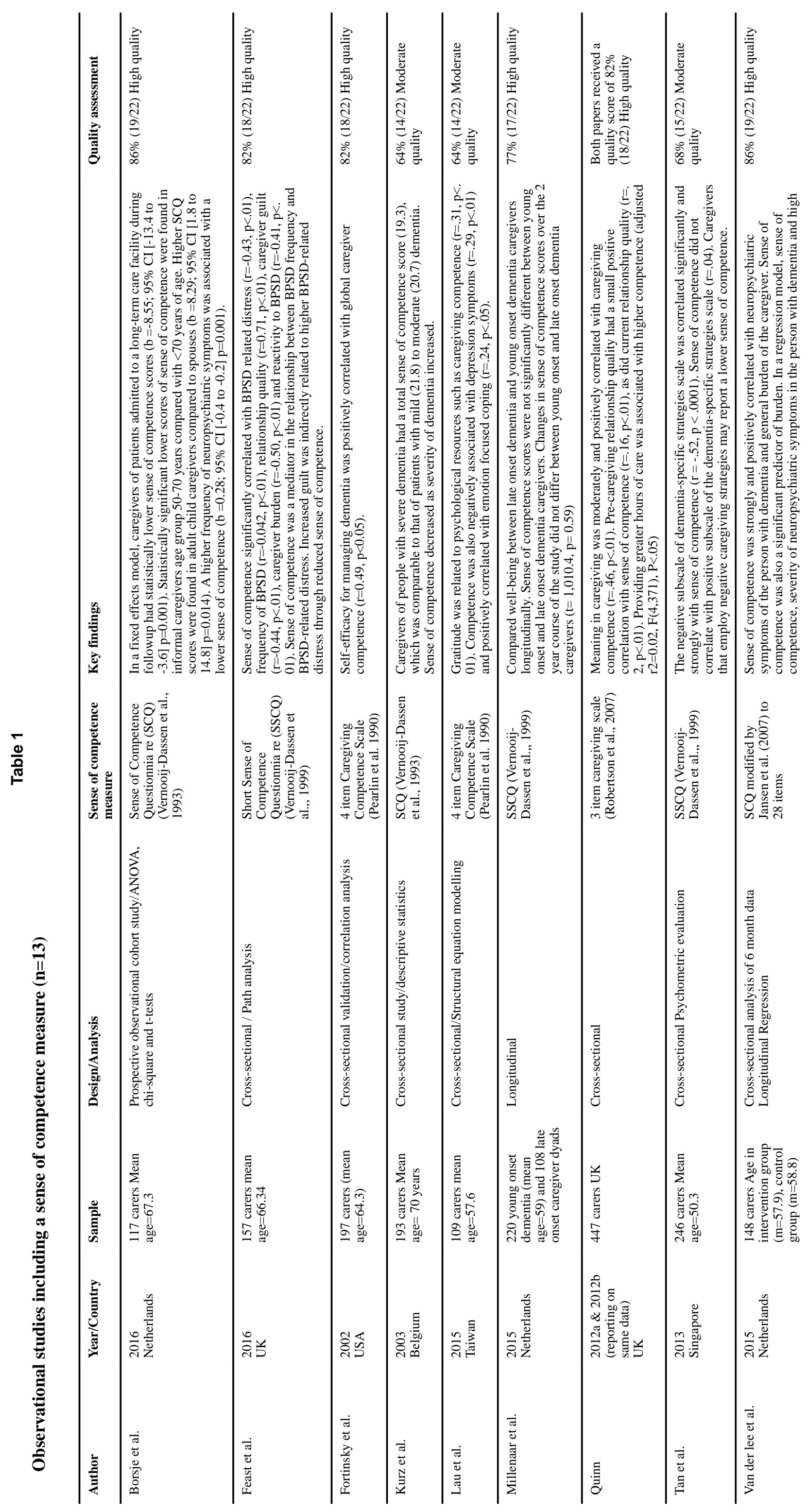




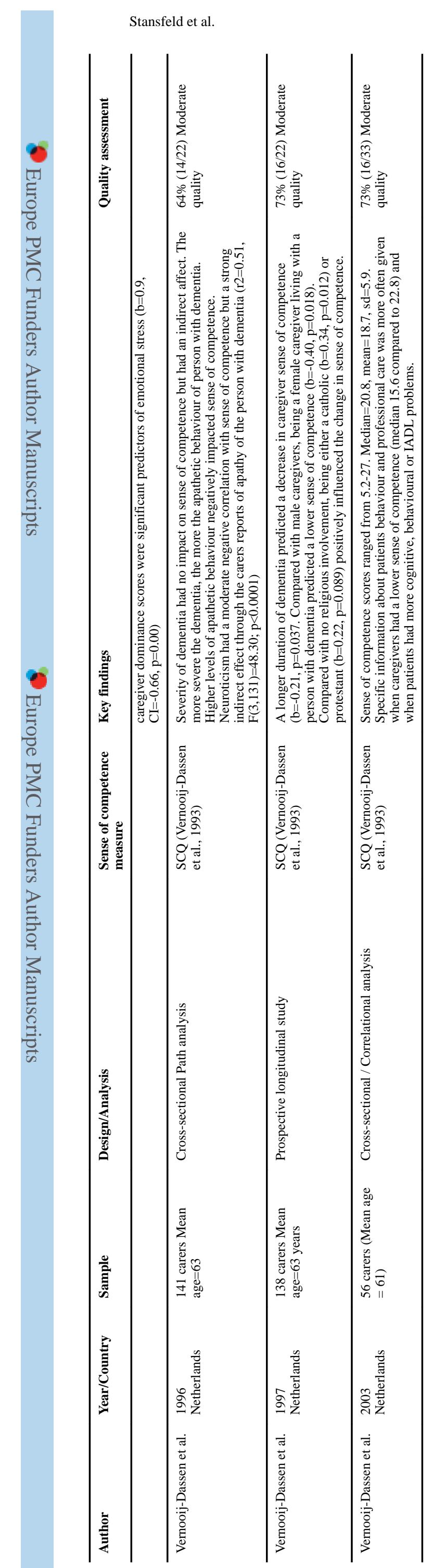

Page 17 


$$
\text { Wi }
$$

